\title{
POLYMERIC SUSPENSIONS VISCOSITY EVOLUTION UNDER A CONSTANT ELECTRIC FIELD
}

\author{
Ahmed Zelifi*1 and Lounis Mourad ${ }^{2}$ \\ ${ }^{1}$ Département d'Hydraulique, Faculté d'Architecture et de Génie Civil. \\ Université des Sciences et de la Technologie d'Oran Mohamed Boudiaf, USTO-MB, \\ BP 1505, EL M'naouer, 3100 Algérie \\ ${ }^{2}$ Département des Sciences, Faculté de Technologie. Université de Khemis Meliana, \\ Algérie \\ *Corresponding author: Zelifi Ahmed \\ ahmed.zelifi@univ-usto.dz
}

(Received March 2018 - Accepted July 2019)

\begin{abstract}
Zelifi, A. and Mourad, L. 2019. Polymeric suspensions viscosity evolution under a constant electric field. Lebanese Science Journal. 20(2): 272-284.

The present paper illustrates the effect of the coupling of an electric field with a shear field on a suspension ER. When the suspensions are simultaneously under flow and under the influence of a low electric field organized into packed lamellar formations, the shear stress increases with the increase of the higher polarisable particle concentration both in the electrostatic and hydrodynamic forces. In the absence of an electric field, the flow, alone, produces no segregation.The curves obtained after analyzes illustrate the changes on the shear viscosity under the simultaneous effect of an electric field and shear rate of the three suspensions study. We also observed the appearance of a white foam layer at the experimental apparatus which results in the electrochemical phenomenon due to some values of electric field. The latter can be exploited for a possible further research.
\end{abstract}

Keywords: Carbopol 940, Electro Rheological, viscosity, Direct Current Electric field, Suspension.

\section{INTRODUCTION}

The electro rheological response defined as dramatic in rheological properties of a suspension of small particles due to the application of a large electric field transverse to 
the flow direction. The structure is altered by the field induced formation of fibrous aggregates aligned with the electric field. These effects are both rapid and reversible. The observation of a large effect was first reported by (Winslow, Willis.1949), analogous to Carbopol 940 in our solution dielectric. In fact, the most commonly observed chains are not single-file lines of particles but are columnar clusters of particles or columns bridging electrodes, bearing diameters many times greater than the particles. In the electro rheological fluids, applied shear deformations induce stresses due to two types of interactions among the dispersed electro rheology particles (Seo, Choi et al. 2012): electrostatic interactions dominate the hydrodynamic interactions to form a chain-like structure of the dispersed particles at low shear rates, while hydrodynamic interaction can break the mesostructures at high shear rates. The electro rheology -active suspensions exhibit a residual shear viscosity that resists disruption of the aligned mesostructures by shear forces at low shear rates but start to flow at higher shear rate. The aligned mesostructures are partly destroyed, thereby initiating flow, at higher shear rates, which the mesoscale structures rebuild afterwards. The shear stress observed after reformation decreases because the structures reform incompletely (Seo, Choi et al. 2012). The resulting change in viscoelasticity properties has been utilized in application such as shock absorbers, clutches, brakes, actuators, artificial joints and robotic controls (Seo, Choi et al. 2012, Bohon, Krause. 1998, Papadopoulos. 1998, Akella, Cutkosky. 1995, Chol, Chol. et al. 1998 ) it is worth noting that certain applications, such as active dampers, involve loading conditions below the yield stress or oscillations with small amplitudes, the Bingham plastic assumption, which works reasonably well with high strain rates below the yield point (Kamath,Werely.1997) for these remarkable properties, electro rheology becomes one of the most important actuators used in smart materials and structure (Leng, Liu. 1995), Being developed within these late years (Bullough, Jokeman. 1998, Takesue, Zhang et al. 1998) . The rheological properties of electro rheology are complex. They depend on various factors. The most important one is the external electric field; The electro rheology 's behaviour is the same as Newtonian fluids; whereas with an electric field, the appeared properties of electro rheology 's are the same as Bingham plastic fluids; Gamota and Filisko (Gamota, et Filisko. 1991), investigated the dynamic properties of electro rheology. They found that electro rheology had various stress responses such as viscosity, linear elasticity, non-linear elasticity, plasticity and all of these responses were affected only by the electric field strength. The reorientation of dispersed particles whose initial random distribution transforms into fibrillated structure result in the change of shear viscosity to a higher value. The homogeneous solutions of liquid crystals are sometimes added to improved colloidal stability of the dispersed particles to enhance ER activity (Sohn, Sung. 2002). The uniform electric fields are usually applied for suspensions which are sandwiched between two parallel plate electrodes (between the two cylinders). But the electric forces acting on the particles depend on the local field intensity. In fact, as it was confirmed in previous papers (Kamibayashi, Ogura et al. 2008), the column formation of particles is enhanced in the electrodes honeycomb pattern and this serves to increase the dynamic viscosity of the electro rheology suspensions. The mechanisms of the electro rheology effect were explained by several ways including the dipole by the electric constant mismatch between 
the carrier fluids and the particles, Marshall et al. (Kamibayashi, Ogura et al. 2008). Few research works concluded on how the particle shape influences the electro rheology effect. However, it is well known that the dielectric properties of a heterogeneous system largely depend on the geometry of the dispersed particles. This article examines the results of an extensive study on the discussion and justification of the reversible reaction of the electrorheological activity related to changes in the dynamic viscosity under electric field intensity application. Furthermore, we provide a detailed presentation of the viscoelastic properties of our samples with three different concentrations under DC electric field intensity.

\section{MATERIALS AND METHODS}

\section{Materials}

Steady shear rheological measurements were performed using concentric cylinder geometry equipped with low-voltage generator [Phywe] .The electric field was applied transversely to the direction of shear (namely across the concentric cylinders) each cylinder was insulated from the rest of the rheometer. The annular gap between the cylinders was $1.64 \mathrm{~mm}$ [Haake]. The electric field was applied to the gap by grounding the outer cylinder and connecting the inner cylinder to a low-voltage source. All measurements are conducted at $21^{\circ} \mathrm{C}$, the flow curves for each electro rheology fluid were determined in the controlled shear rate mode then the dynamic viscosity was measured. An experimental apparatus and the devices are shown in (Figure 1).

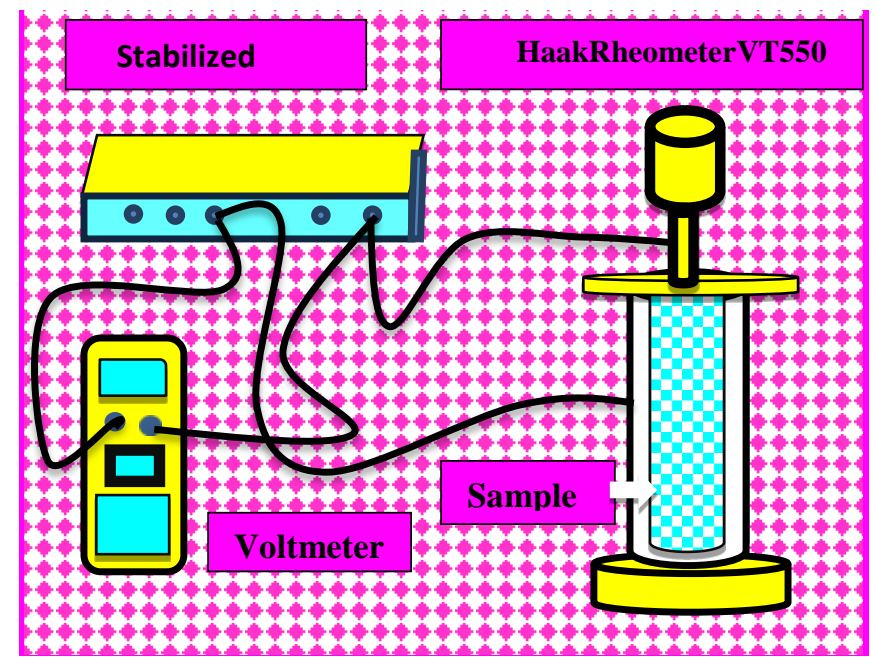

Figure 1. Apparatus diagram scheme used for measurement of dynamic properties.

The measurement was made at a voltage frequency of $50 \mathrm{~Hz}$ and the viscosity through the suspensions was measured in a shear rate range between 6.45 and $645 \mathrm{~s}-1$. The electric field strength (ranging from 0.0 to $19 \mathrm{kV} / \mathrm{m}$ ) was kept constant during the performance of 
each shear rate sweep; the suspension was loaded into the Haake VT550 rheometer, and the electric shear rates were applied. A positive terminal has been applied to the inner cylinder shaft and the outer cylinder to a positive terminal and these two terminals will be connected to the electrical generator. The Direct Current voltage is applied by a technique of frictionless contact at the Haake rheometer VT550, and calibrated power source was used to supply the desired voltage.

Figure 1 shows a schematic diagram of the apparatus used for the measurement of the dynamic viscosity properties the Carbopol 940 the prover polymers in pharmaceuticals, (B, F. Goodrich Cleveland, OH, USA (David B. Braun., Meyer R, Rosen), in addition the Carbopol was added to the micro emulsion at up $2 \mathrm{wt} \%$.

\section{Methods}

The aqueous dispersion is obtained on the basis of a powder mixture of Carbopol 940 and distilled water to obtain a fluid suspension of different concentrations (1.0, 1.5 and 2.0) wt. \% by weight and which were chosen according to both the operating range of the rheometer VT550 and previous studies approaches.

The values (1.0, 1.5 and 2.0) of the concentrations in weight were chosen on the basis of the classic viscometer of the type: rotating cylinders (Couette flow) Rheometer VT 550 (HAAKE ViscoTester 550) [Haake], for concentration values above $2.0 \%$ by weight the fluid will become too viscous and this prevents the measuring sensor from operating (MV / DIN) [Haake]. Similar proportions have been studied by the authors Anuvat Sirivat, Rathapon Asasutjarit et Panida Vayumhasuwan.

To refine the Carbopol 940 powder in order to get a narrow distribution of particle size, we manually crushed powder through the porcelain mortar, to prevent large particles significantly reduce the breaking strength of the electro rheology fluid. For the preparation of Carbopol powder, stock solution 940 is dried at a temperature of $50^{\circ} \mathrm{C}$, so the procedure is to weigh an exact amount of $(1 \mathrm{~g})$ of dried powder of Carbopol 940 in a volume of $100 \mathrm{ml}$ of distilled water. Then this mother solution is put under stirring for 24 hours via a magnetic stirrer. To get the recommended dispersion homogeneous mixture will be established to stirring at $5000 \mathrm{rpm}$ to drive out any air trace. This suspension is stored in tightly closed containers to prevent water evaporation. From the mixture are carried appropriate dilutions for our research namely concentrations of 1.0, 1.5 and $2.0 \mathrm{wt} \%$. The $\mathrm{pH}$ of the samples prepared in distilled water has a 6 average. All suspensions were kept under control for at least 24 hours at ambient temperature before the experimental procedure rheological measurements to be held. The homogeneity stability will be much improved if we actually inject the mother solution as the additive $\mathrm{NaOH}$. The so-called positive electrorheological materials have remarkable rheological properties under application of an electric field to direct current. 


\section{RESULTS AND DISCUSSION}

We have limited our research to the applications of the electro rheology effect to low electric field DC voltage in this section. The phenomenon of the shear viscosity dynamic in electro rheology suspensions were commonly represented by the Bingham constitutive equation. The Bingham model can be used to describe out suspensions at low concentrations only. The work described above emphasizes the important role the electric current passing through the particle chains, the viscosity enhancement is much larger at low shear rates and the flow becomes shear thinning. For electro rheology suspensions, a constant stress which corresponds to the Bingham yield stress is developed at low shear rate in electric fields. When a DC electric field is applied to the sample, an immediate separation of the concentration of constituents while polarization is observed in the direction of the electric field and the gap between the electrodes were bridged chains of particles. The dispersed particles have a random arrangement to zero electric field, when an electric field is applied, there is a rearrangement of the particles having a chain structure type; the particle of this behavior is similar to that in the electro rheology fluids studies. This chain structure in the electro rheology fluid will disappear quickly after removal of the electric field; the formation of the chain structure in the electro rheology fluid upon application of the electric field will cause the disappearance of the bias force due to the difference in permittivity within the electro rheology fluid of the particles and the conduction phenomenon simultaneously. Under the effect of shear rate and electric field shown by (Figures. 3-4), the measured values of the shear viscosity of the electro rheology fluid are highly variable for three suspensions for biasing same duration imposed by the electric field. These phenomena are in accordance with the measurement of polyaniline samples, which compares favorably with that of Mac-Diarmid and al. (Choi. Cho. 1999).

The polarization of the forces between the particles increases with the effect of the electric field intensity and the particles concentrations in certain regions of figures 27. Thus resulting in large variations of the shear viscosity because of progressive rupture of periodic structure with increasing shear rate, the flow of electrified suspension becomes shear thinning. In figure 2 , the curves have a trend of bells and the shear rate value is equal to $38.43 \mathrm{~s}-1$ in an electric field $\mathrm{E}=(0-11) \mathrm{kV} / \mathrm{m} \mu$ measuring increases (125 to 350) $\mathrm{mPa}$.s and the shear viscosity of the aqueous Carbopol dispersions decrease with the electric field, over an electric field to $\mathrm{E}=(11-17) \mathrm{kV} / \mathrm{m}$ thus $\mu$ the measure decreases to (350 to 150) mPa.s. The results in (Figure 3a) show that these $\mu$ versus plots reflect that electrified dispersions exhibit a shear thinning behavior that can be easily associated with degradation of the induced structure in the electro rheological fluid.

In figure 3, three regimes are presented, for example by taking the rate of shear value is $38.43 \mathrm{~s}-1$ : the first regime measures pass (350-590-250) $\mathrm{mPa}$.s for $\mathrm{E}=(0-3-5)$ $\mathrm{kV} / \mathrm{m}$; for the second regime, the $\mu$ values varied from (250-850-425) mPa.s for values of $\mathrm{E}=(5-8-13) \mathrm{kV} / \mathrm{m}$. 


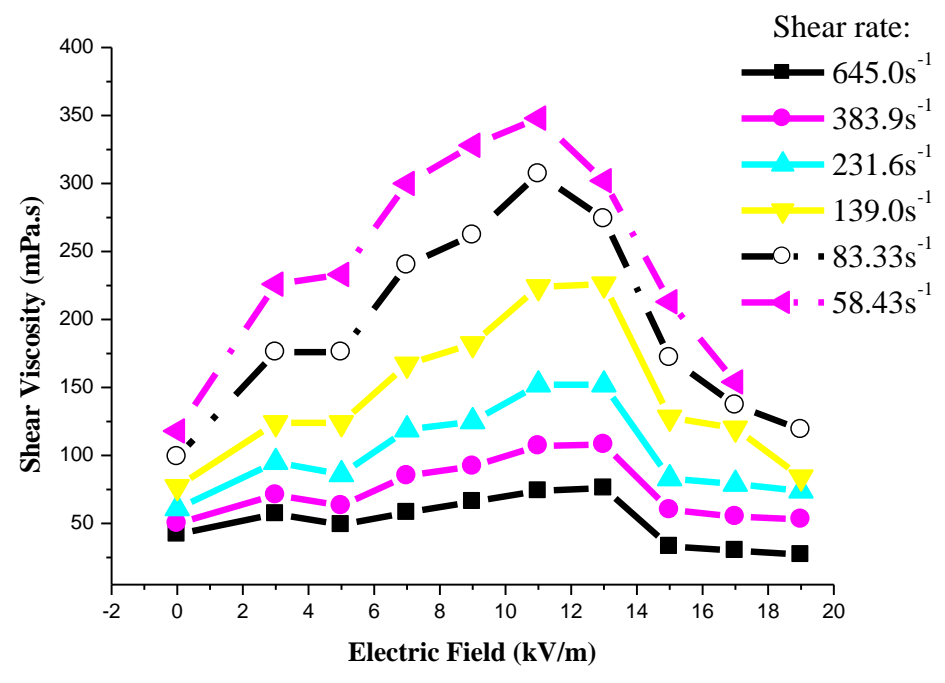

Figure 2. Variations of the shear viscosity vs applied electric field for value shear rate (suspension of 1.0wt \%).

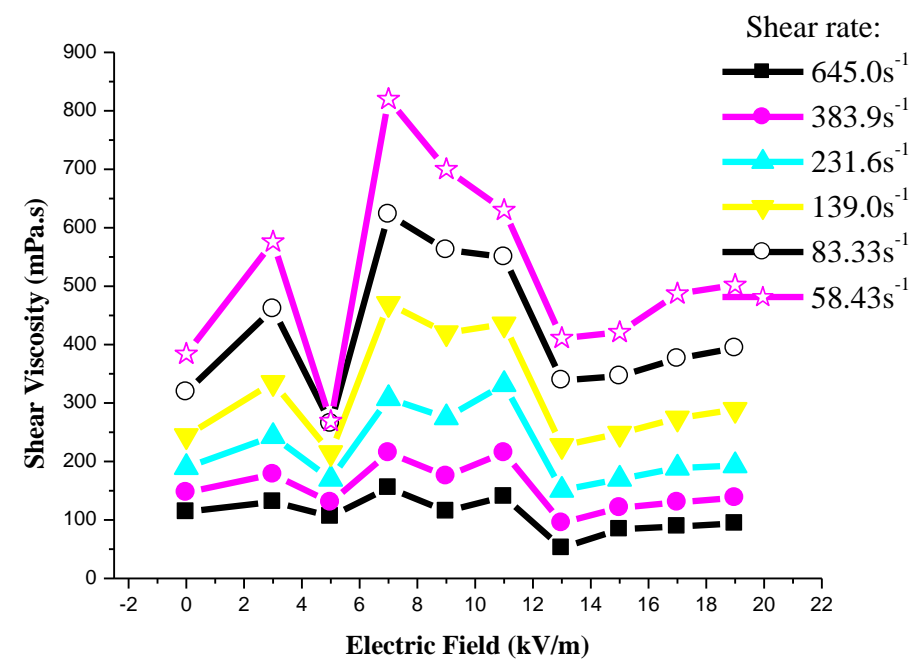

Figure 3. Variations of the shear viscosity vs applied electric field for value shear rate (suspension of $1.5 \mathrm{wt} \%$ ).

These sudden ruptures are the consequences of change under intense shearing effect of the electro rheology fluid. Finally, concerning the last regime, we note that the variations are very low $\mathrm{E}=13 \mathrm{kV} / \mathrm{m}$. Reduced dynamic viscosity induced by the field result of late migration of particles towards the electrode (solid-liquid) where aggregate formation and no influence of the electric field in this situation and the suspension effect in the electro rheology, probably due to Quincke rotation on the behavior of suspension 
Thus the figure 4 has two phases, the first of the $\mu$ measures are (900-1200-390) $\mathrm{mPa} . \mathrm{s}$ to an electric field $\mathrm{E}=(0-5-7) \mathrm{kV} / \mathrm{m}$, the escalation of the curves is favored by the simultaneous effect of the electric field and shear rate of the suspension; the decrease in viscosity is due to the weakness of the strength of polarization and electrophoresis phenomenon; the second phase, the measures $\mu$ back of (390-550) mPa.s to an electric field $\mathrm{E}=(7-19) \mathrm{kV} / \mathrm{m}$, the viscosity variation is linear in this case for the attachment of the chain polymers. According to the analysis mentioned above, the best results from experimental viscosity measurements are shown in (Figure $3 \mathrm{~b}$ ) especially for values of $\mathrm{E}$ $=(5$ to 13$) \mathrm{kV} / \mathrm{m}$. The conduction properties of solid-liquid phase largely influences the intensity of the electro rheology effect, that is to say, energy-amplifying power appears between the control parts (electric) and controlled (mechanical).

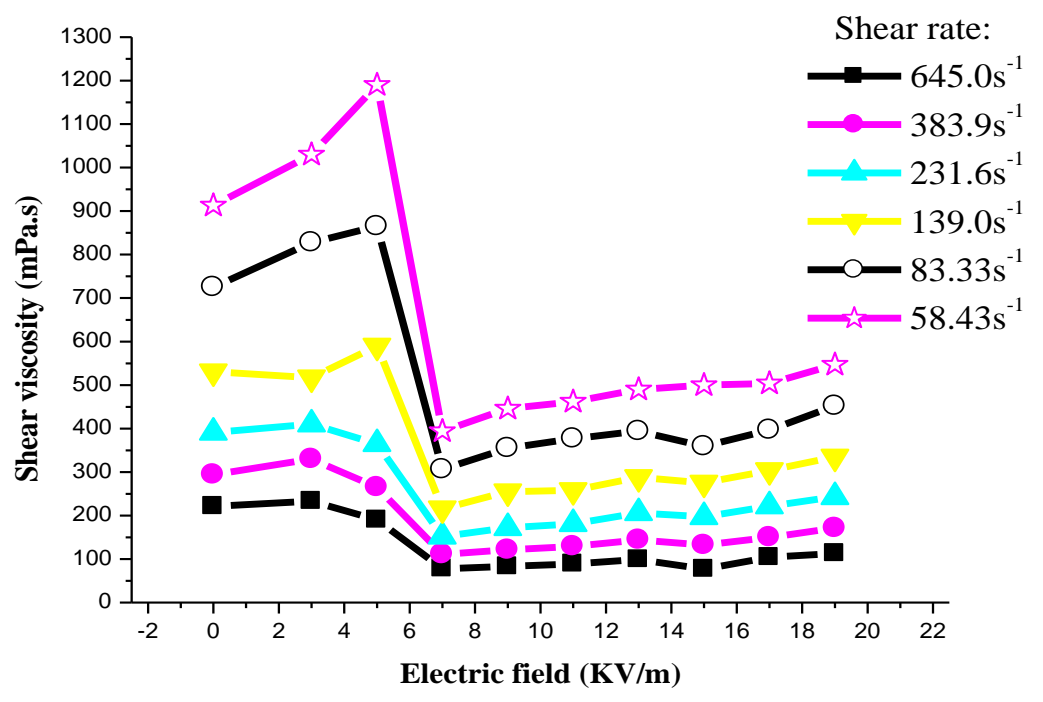

\section{Figure 4. Variations of the shear viscosity vs applied electric field for value shear rate (suspension of $2.0 \mathrm{wt} \%$ ).}

The study of the three suspensions of concentrations respectively 1.0, 1.5 and $2.0 \mathrm{wt} \%$ shows that the experimental curves obtained (Figure 2, 3 and 4) do not have the same regime following the application of the same electric field. It is then noted that the high dose Carbopol 940 suspension under a low velocity gradient (= 58. 43s-1) has better dynamic viscosity values for an electric field (E) of less than $9 \mathrm{kV} / \mathrm{m}$ (see Table 1). However, for large values of the velocity gradient (=645 s-1) the results of the dynamic viscosities are very low, probably due to the consequences of heat diffusion and formation of aggregates chains (solid-liquid) which appear at the end of flow. Knowing then the aspect of the intense friction at the wall of the internal cylinder of the rheometer which creates the phenomenon of kinetic heating (appearance of the effect of foam), it is necessary to apply flow velocities under a large number of Reynolds to obtain better dynamic viscosity values. 
Table 1. Outstanding values of dynamic viscosity versus electric field.

\begin{tabular}{|c|c|c|c|c|c|c|}
\hline \multirow{2}{*}{$\begin{array}{c}\text { Shear rate } \\
\left(\mathrm{s}^{-1}\right)\end{array}$} & \multicolumn{5}{|c|}{ Direct Current: E (kV/m) } & \multirow{2}{*}{$\begin{array}{l}\text { Suspension } \\
\text { fluidique }\end{array}$} \\
\hline & 5 & 7 & 9 & 11 & 13 & \\
\hline 58.43 & & & & $350 \mathrm{mPa} . \mathrm{s}$ & & \multirow[t]{2}{*}{$1.0 \mathrm{wt} \%$} \\
\hline 645.0 & & & & $75 \mathrm{mPa} . \mathrm{s}$ & & \\
\hline 58.43 & 300mPa.s & & $800 \mathrm{mPa} . \mathrm{s}$ & & $400 \mathrm{mPa} . \mathrm{s}$ & \multirow{2}{*}{$1.5 \mathrm{wt} \%$} \\
\hline 645.0 & $100 \mathrm{mPa} . \mathrm{s}$ & & $150 \mathrm{mPa} . \mathrm{s}$ & & $50 \mathrm{mPa} . \mathrm{s}$ & \\
\hline 58.43 & $1200 \mathrm{mPa} . \mathrm{s}$ & $400 \mathrm{mPa} . \mathrm{s}$ & & & & \multirow{2}{*}{$2.0 \mathrm{wt} \%$} \\
\hline 645.0 & $200 \mathrm{mPa} . \mathrm{s}$ & $50 \mathrm{mPa} . \mathrm{s}$ & & & & \\
\hline
\end{tabular}

As the electric field strength increases, the electrostatics becomes dominant over the hydrodynamic forces (Kawai, Ikazaki.2002, Davies, Blagbrough. 1998). After the rotation of the particle, the interparticle force becomes weaker as the external surface charges on the particle which creates a local electric field just opposite the external electric field which leads to the negative electro rheology effect. Therefore, shown in (Figure 6) the largest increases in suspension viscosity occur at small shear rate and large field strengths (Parthasarathy, Klingenberg. 1996). The viscosity enhancement is much larger at low shear rates and the flow becomes shear thinning. Indeed although a study of the structure of the suspensions is not the aim of this paper (detailed studies can be found in refs) (Espin, Plocharski. 2007). Thus in this suspension of $2.0 \mathrm{wt} \%$ a very small change in the shear rate (0-100) s-1 during the measurement can cause a large change in viscosity. We note that from the value shear rate $625 \mathrm{~s}-1$ in the congealed state region, the resistivity decreases linearly with increasing the applied field.

The results 4 of the graphs illustrated in three (Figures 5, 6 and 7) clearly show that $\mu$ changes in shear rate function under the effect of the electric field $(E=2-4 \mathrm{kV} / \mathrm{m})$ increase in general. The mean changes from $\mu$ spend (50 to 500) $\mathrm{mPa}$.s during the solicitation period and evolution of $\mu$ is influenced by increases in the electric field and the shear rate values (220-650) s-1, the graphs configuration is linear, as there is a large deposit of électropherique electro rheology fluid: see (Figure 5). Obviously, $\mu$ increasing (170-2000) $\mathrm{mPa}$.s depends on the concentration of the suspension and low variation to an electric field, where $\mathrm{E}=2$ and $4 \mathrm{kV} / \mathrm{m}$ require special attention of the electrorheological fluid: see (Figure. 3e). Finally, with regards to the analysis of the curves in (Figure 6); through experiment, we also observed that the shear viscosity was proportional to the electric strength (Ronald,H.W. Hoppe, and William,G.Litvinov.2011), the $\mu$ value was $3000 \mathrm{mPa} . \mathrm{s}$ at a shear rate $(50 \mathrm{~s}-1)$ at an electric field of $3 \mathrm{kV} / \mathrm{m}$ and beyond this biasing is observed a presence of electrochemical phenomenon which generates the foam effect (see Figure 8). 


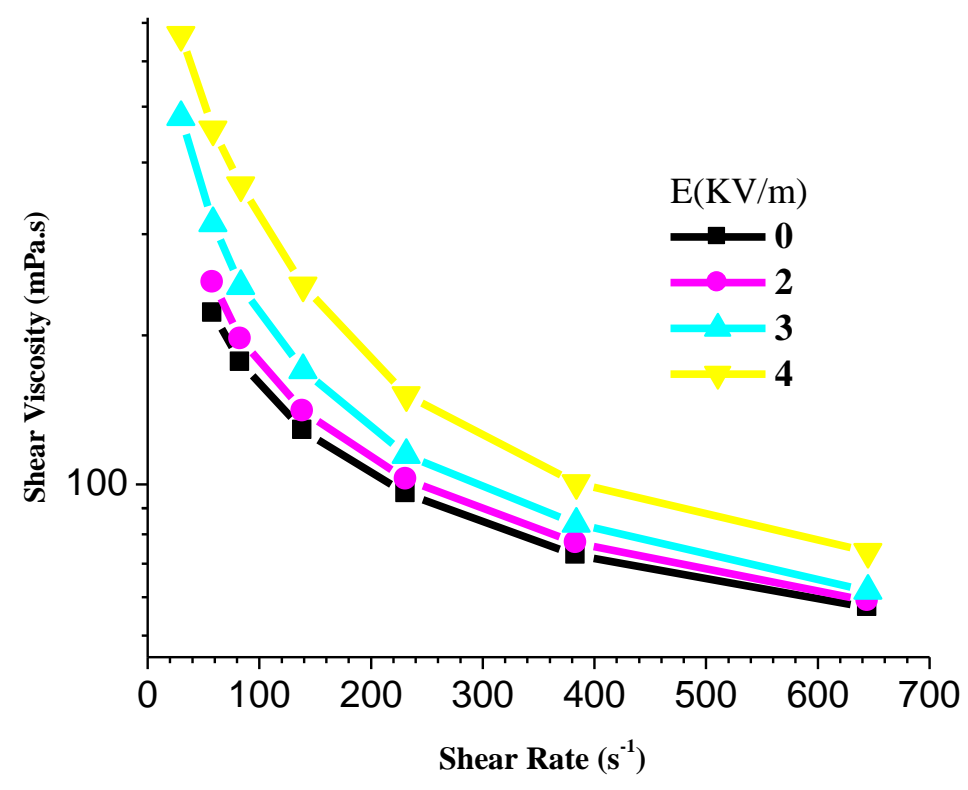

Figure 5. Variations of the shear viscosity vs applied shear rate for different electric field values: (suspension of 1.0wt \%).

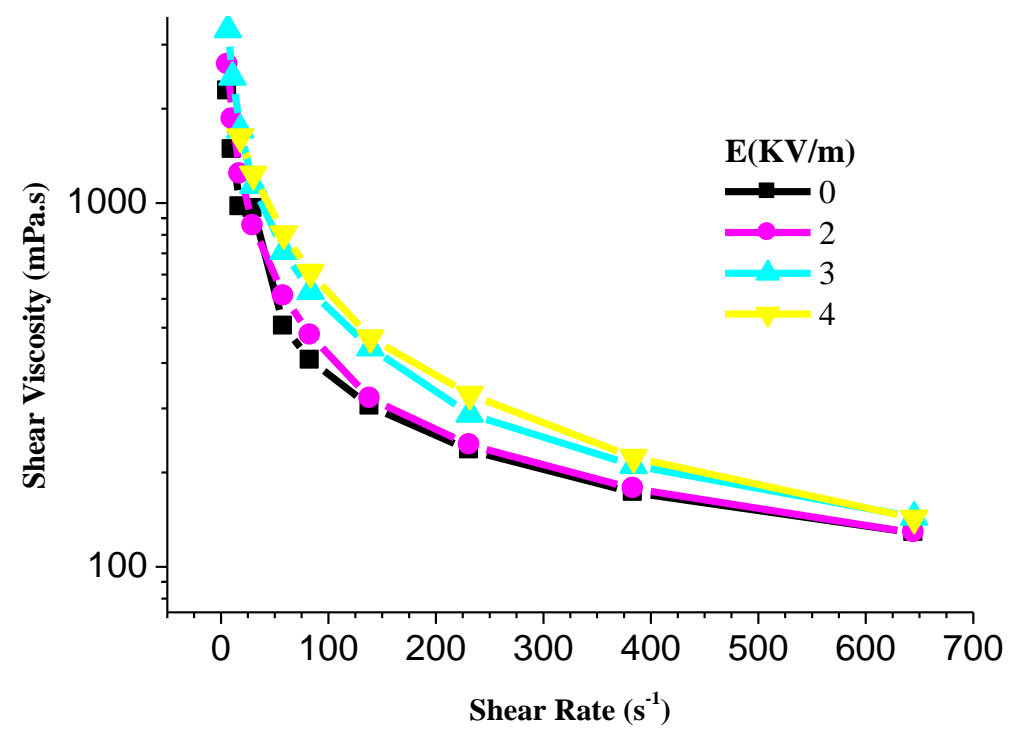

Figure 6. Variations of the shear viscosity vs applied shear rate for different values electric field: (suspension of $1.5 \mathrm{wt} \%$ ). 


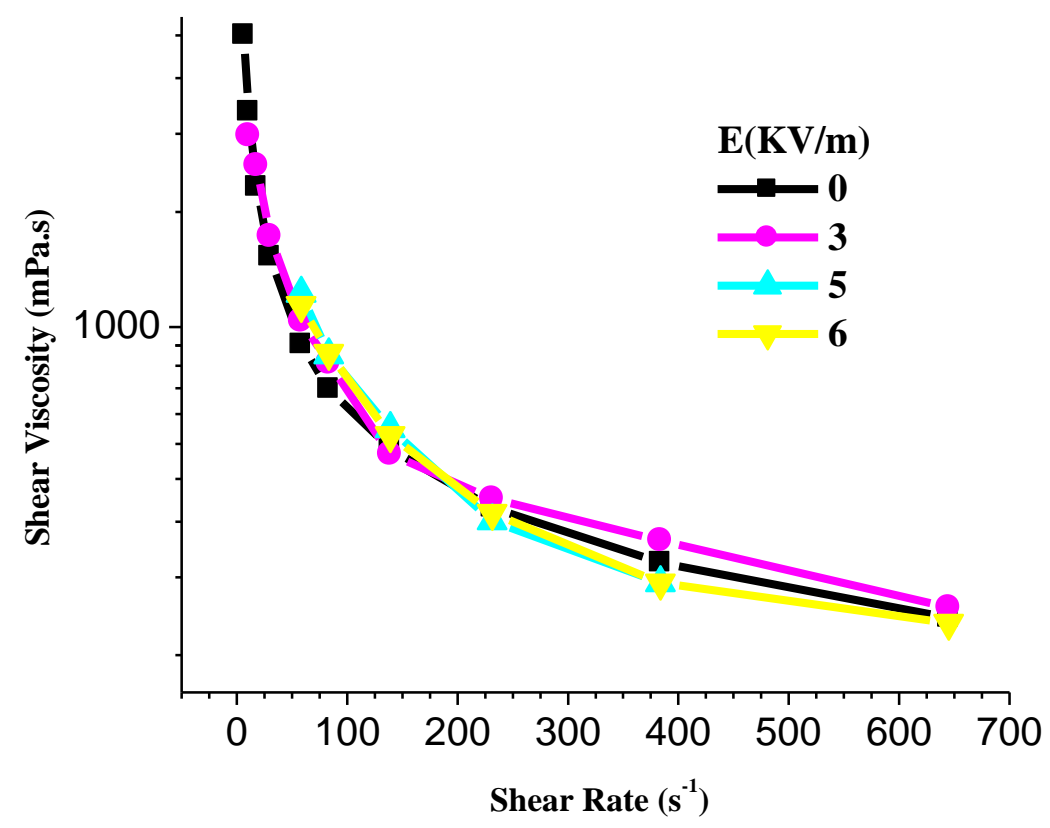

Figure 7. Variations of the shear viscosity vs applied shear rate for different values electric field: (suspension of $2.0 \mathrm{wt} \%$ ).

This characteristic is due to the viscous forces of the electro rheological fluid, disturbed by the dual effect of both electrical and thermal conduction (Ohm's law) (Fourier's law).

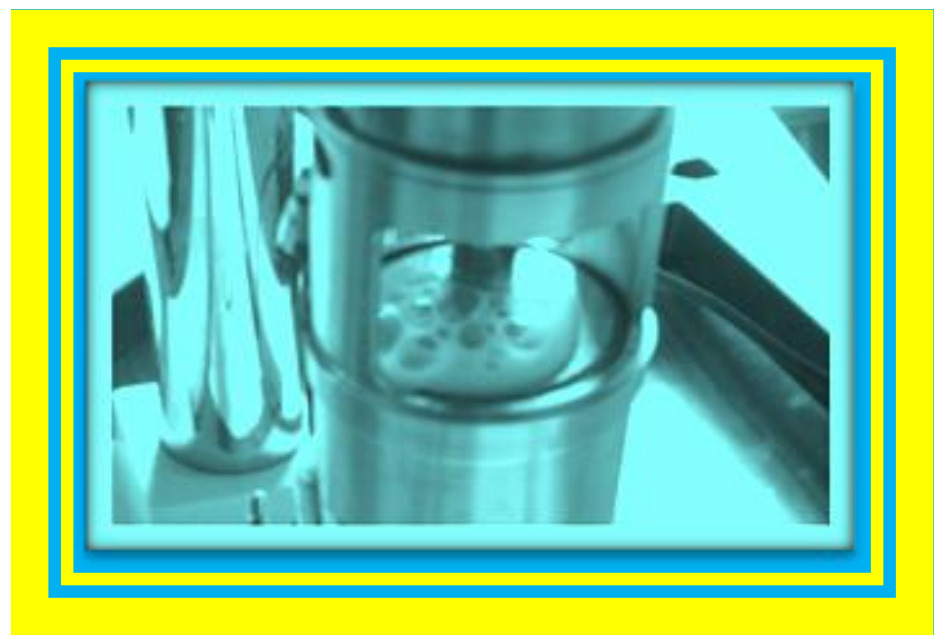

Figure 8. Formation of foamy white mass due to the electrochemical effect during the application of the electric field. 
According to the specified VT 550 rheometer; it is observed that the electrorheological properties of the suspension (Figure 3 and Table 2) are very close to the interval $(\mathrm{E}=2-3) \mathrm{kV} / \mathrm{m}$ and all those which have a range of velocity gradient of the VT 550 rheometer. Therefore, the Carbopol 940 gel structure possessing predominantly elastic solid behavior and showing extensive entanglements between different polymer chains could act as a fine mesh impeding the diffusing's movement.

Table 2. Variations in dynamic viscosity $(\Delta \mu)$ as a versus of the electric field.

\begin{tabular}{|c|c|c|c|c|c|c|c|c|c|}
\hline \multirow{3}{*}{$\begin{array}{l}\text { Shear rate } \\
\qquad\left(\mathrm{s}^{-1}\right)\end{array}$} & \multirow{2}{*}{\multicolumn{3}{|c|}{$\begin{array}{c}\text { Suspension fluidique }(\mathrm{d}) \\
\text { variation of electric current: } \\
\Delta \mathrm{E}(\mathrm{kV} / \mathrm{m})\end{array}$}} & \multirow{2}{*}{\multicolumn{3}{|c|}{$\begin{array}{c}\text { Suspension fluidique }(\mathrm{e}) \\
\text { variation of electric current } \\
\Delta \mathrm{E}(\mathrm{kV} / \mathrm{m})\end{array}$}} & \multirow{2}{*}{\multicolumn{3}{|c|}{$\begin{array}{c}\text { Suspension fluidique (f) } \\
\text { variation of electric current } \\
\Delta \mathrm{E}(\mathrm{kV} / \mathrm{m})\end{array}$}} \\
\hline & & & & & & & & & \\
\hline & $0-2$ & $2-3$ & $3-4$ & $0-2$ & $2-3$ & $3-4$ & $0-3$ & 3-5 & $5-6$ \\
\hline 6.45 & - & - & - & 370 & 400 & - & - & - & - \\
\hline 10.7 & - & - & - & 300 & 520 & - & - & - & - \\
\hline $\begin{array}{l}17.93 \\
\end{array}$ & - & - & - & 225 & 390 & - & - & - & - \\
\hline 29.93 & - & - & - & 157 & 255 & 80 & - & - & - \\
\hline 58.43 & 26.0 & 60.5 & 130 & 97 & 182 & 86 & 117 & 160 & - \\
\hline 83.33 & 16.0 & 42.5 & 110.5 & 64 & 139 & 72 & 103 & 37 & - \\
\hline 139 & 10.0 & 24.0 & 64.0 & 14 & 110 & 26 & - & 73 & - \\
\hline 231.6 & 5.7 & 10.5 & 30.5 & 7 & 477 & 35 & - & 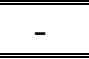 & - \\
\hline 383.9 & 3.5 & 6.4 & 15 & 4 & 26 & 10 & - & - & - \\
\hline 645 & 1.6 & 2.6 & 11.1 & 0 & 13 & - & - & - & 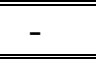 \\
\hline
\end{tabular}

Therefore, the Carbopol 940 gel structure possessing predominantly elastic solid behavior and showing extensive entanglements between different polymer chains could act as a fine mesh impeding the diffusing's movement. The fluid considered is viscoelastic and the quantity of Carbopol 440 used in this suspension is very compatible since the experimental data obtained are better parametric electrorheological qualities.

Naturally, for given applied forces, the greater the intermolecular forces and the greater the viscosity of the fluid; therefore, the lower the amount of fluid displaced. However, for the other suspensions with low viscosity properties and too viscous (see Table.2), the results obtained show that the simultaneous effects of the viscous heating of the suspension and the strong flows due to speed gradients; the macromolecules can be oriented or deformed by preventing the stability of the homogeneity of the fluid while generating the phenomenon of the appearance of the aggregate chains and diffusion of heat and mass. We note that the value of the electric current $\mathrm{E}=3 \mathrm{kV} / \mathrm{m}$ presents an optimal value of measurement during the experiments carried out on our suspensions. 


\section{CONCLUSION}

As shown in this paper, we succeeded in getting information relevant to that of the shear viscosity in electric field strengths. The steady-state rheological properties of electro rheology suspensions containing Carbopol 940 particles have been studied. Two important conclusions can be drawn: First, the dispersed phase particle concentration and electric field strength, on the basis of the rheological observations, we can conclude that, when the polymer concentration of Carbopol 940 is close to $1.5 \mathrm{wt} \%$, the viscoelasticity and flow properties of the micro emulsions are suitable to ensure an adequate performance for a better study of the results of shear viscosity. We can therefore conclude that the $1.5 \mathrm{wt} \%$ suspension present as an element for the study of continuous electric field electro rheology fluid. The results of the present study show the following relationships between the molecular structure and the electro rheology effect of liquid. Second, the decreases in the shear viscosity values observed at the shear rate of $645 \mathrm{~s}-1$ are at the origin of the increase of the electric field at the time of the formation of the aggregates: solid- liquid separation, except in the case of the suspension $2.0 \mathrm{wt} \%$, a new phenomenon that appears namely with the effect of foam or edge effect. Increases in Carbopol 940 concentrations induced the preparations in exhibit a more elastic solid behavior. The latter will be discussed in more details in a future publication. The observed energy dissipation at the two coaxial cylinders during the solicitation period and the presence of aerodynamic effects may be considered additional elements to decrease the shear viscosity. For best results of the shear viscosity during the experimental procedure we should take into consideration the number of Brinkman to completely avoid the Joule effect, while controlling the power output during periods of application of the electric Direct Current field.

\section{REFERENCES}

[Haake] www.The Viscometer System, HAAKE: (Thermo Scientific Viscometers [PDF], $\mathrm{pp}(6-8))$.

[Phywe] https://www.techno-sciences.fr/ Ref.: 13506-93

Akella, P.N and Cutkosky, M.R. 1995. Contact transition control with semi active soft fingertips. IEEE Transactions on Robotics and Automation .11(6): 859-867.

Bohon, K. and Krause, S. 1998. An electrorheological fluid and siloxane gel based electromechanical actuator: Working toward an artificial muscle. Journal of Polymer Science Part B Polymer Physics. 36(6): 1091- 1094.

Bullough, W.A. and Jokeman, N.1998. Limited Motion Electrical Actuation Systems, IEE. Colloq. 5Digest ${ }^{\circ}$. 494.

Chol, S.B. Chol, Y.T. Chang, E.G. Han, S.J. and Kim, C.S. 1998. Publisher not identified. Mechatronics. 8: 143.

D. R. Gamota and F. E. Filisko. 1991. Dynamic mechanical studies of electrorheological materials: Moderate frequencies. Journal of Rheology. 35(3): 399.

David B. Braun., Meyer R, Rosen, Rheology Modifiers, Handbook practical use and application, pp. 72-86, William Andrew publishing Norwich, New york, USA. 
Davies, J.L. Blagbrough, I.S. and Staniforth, J.N. 1998. Electrorheological behavior at low applied electric fields of microcrystalline cellulose in BP oils. Chemical Communications. 19: 2157-2158.

Espin, M.J. and Plocharski, J. 2007. Effect of pollution on the interfacial properties of electrorheological fluids. Colloids and Surface A: Physicochemical and Engineering Aspects. 306(1-3): 126-136.

Kamath, G.M and Werely, N.M. 1997. Smart Materials and Structures. A nonlinear viscoelastic - plastic model for electrorheological fluids. 6(3): 351.

Kamibayashi, M., Ogura, H. and Otsubo, Y.2008. Shear-Thickening Flow of Nanoparticle

Leng, J.S. Liu, Y.J. Dul, S.W. and Wang, D.F. 1995. Applied. Composite Materiales. Active vibration control of smart composites featuring electro-rheological fluids. 2(1): 59-65.

M. Parthasarathy and D. J. Klingenberg. 1996. Electrorheology: Mechanisms and models. Materials Science and Engineering; R: Reports. 17(2): 57-103.

Papadopoulos, C.A. 1998. Brakes and clutches using ER fluids. Mechatronics. 8(7): 719.

Ronald, H.W. Hoppe, and William, G. Litvinov. 2011. Modeling, Simulation and Optimization of Electrorheological. Fluids Handbook of Numerical Analysis. XVI, P.G Ciarlet (Edition) Elsevier B. (16): 719-793.

Seo, P.Y. Choi, H.J. and Seo, Y. 2012. A simplified model for analyzing the flow behavior of electrorheological fluids containing silica nanoparticle-decorated polyaniline nanofibers. Soft Matter. 8: 4659.

Seo, Y.P. Choi, H.J. and Seo, Y. 2012. Magneto rheology: Advances and Applications. Langmuir. 28: 3077-3084.

Sirivat, A. Asasutjarit, R. et Vayumhasuwan, P. Pharmaceutical research, 22(12).

Sohn, J.L. Sung, J.H. Choi, H.J. and Jhon, M.S. 2002. Electro rheology of undoped poly (p-phenylene) particle-based suspension. Journal of Materials Science. 37(19): 40574061.

Suspensions Flocculated by Polymer Bridging. Journal of Colloid and Interface Science, 321: 294-301.

Takesue, N. Zhang, G. Furusho, J. and Sakaguchi, R. 1998. Robotics and Automation. Proceedings. IEEE. International Conference on robotics and Automation. 2470.

Winslow, W.M. and Willis, M. 1949. Induced Fibration of Suspensions. Journal Applied Physics, 20: 1137. 